\title{
Availability of biological cancer drugs under research: registration and price in Brazil, Colombia, and Mexico
}

\section{| ${ }^{1}$ Gabriela Bittencourt Gonzalez Mosegui, ${ }^{2}$ Fernando Antõnanzas Villar |}

Abstract: This study seeks to understand biological cancer drug availability through registration and prices of the biological agents used for cancer therapy and authorized for sale in the last 5 years in Brazil, Colombia, and Mexico, comparing the data to those for the United States of America (USA) and Spain. The regulatory agencies' websites were assessed for drugs registered between January 1, 2014, and February 20, 2019. Drug prices were sought in the clerical databases. Prices were also compared using purchasing power parity (PPP). The comparison between the purchasing power (PP) of the three Latin American countries is hampered by market heterogeneity and uncertainty in the data. There is no registration synchronization. The average difference between the launch time in the USA and in the other countries is 1.6 to 2.6 years. The USA has the lowest PPP values, compared to the Latin American countries studied, but higher prices. Differences in registration time reveal issues in drug access in the Latin American countries studied or a lack of equity between countries. The economic effort that these countries make to have access to these supplies is much higher than that of the USA and Spain.

> Keywords: biological products; neoplasm; access to essential medicines and health technologies; products registration; drug price.

\author{
1 Instituto de Saúde Coletiva, \\ Universidade Federal \\ Fluminense. Niterói-RJ, Brasil \\ (gabrielamosegui@uol.com.br). \\ ORCID: 0000-0001-5954-684X \\ 2 Facultad de Economia, \\ Universidad La Rioja, Logroño, \\ La Rioja, España (fernando. \\ antonanzas@unirioja.es). \\ ORCID: 0000-0001-6122-9130
}

Recebido em: 02/05/2020 Aprovado em: 09/09/2020 Revisado em: 07/10/2020 


\section{Background}

Produced in living systems through biotechnological processes, biological drugs or biopharmaceuticals are usually proteins or large molecules of high complexity that are fundamentally different from traditional drugs and are considered synthetic, simpler, and smaller (GRAMPP; RAMANAN, 2013). Some agents in this class (biological) can locate the substance against which they are produced in a highly sensitive and specific manner, marking or destroying tumor cells, stimulating or inactivating receptors and enzymes, and interrupting pathological processes (INTERFARMA, 2013; GRAMPP; RAMANAN, 2013). Their use has revolutionized the treatment of various diseases; these agents have been efficiently employed against cancers, autoimmune diseases, diabetes, hepatitis, and other diseases, increasing the survival of these patients.

For cancer therapy, the aim of biological agent use is to prolong and improve the quality of life of patients coexisting with a disease. The regulatory approval for biological agents is similar to that of other technologies, but clinical trials associated with them often evaluate indirect measures or "substitutes" for efficacy (endpoints), demonstrating their biological activity. Some agents have been authorized quickly but were subsequently removed from the market because their endpoints were not adequate (CHARY; PANDIAN, 2017; DAVIS et al., 2017; KIM; PRASAD, 2015; PUTHUMANA et al., 2018; RAPS, 2018).

Given the complexity of their structures and their production processes, greater investments are needed in the manufacture of biological agents. Research and development $(\mathrm{R} \& \mathrm{D})$ and failure during early stages increase the risks of investment and raises the prices of these products. The escalation in global expenditures on drugs, especially for cancer patients, may be a reflection of the high cost of target therapies that dominate the field (INTERFARMA., 2013).

Even with high efficacy, the costs of biological agents added to other already existing costs significantly affect governments, health systems, and services, threatening the economic sustainability of these institutions (RUIZ et al., 2017). The price of, access to, and expenditure for biological agents are different among countries with high, medium, and low income. The analysis of empirical data on the extent of health regulations of these supplies, whether of registration or prices, can help in understanding their availability in Latin American countries, where 
health systems function differently (DAVIS et al., 2017; PUTHUMANA et al., 2018; SANTANA; LEITE, 2016).

Knowing that "access to drugs" is a multidimensional category (ÁLVARES et al., 2017; FERRARIO, 2017; WHO, 2008) defined in different ways, in this study two elements to understand the availability of biological drugs in different markets were chosen: synchronicity of time available in the market and a price that allows the differences between health systems not to be an inconvenience for drug consumption. For a drug to be available in a country, it must be registered with the health agency. If the registration time is similar in all countries, there are no issues regarding access or equity, in comparative terms. A price discrimination policy could increase access so that countries with lower incomes have biological agents with lower prices. This is the general form of Ramsey's inverse elasticity rule (SHEPHERD, 1992).

Given this scenario, the aim of this study is to examine drug availability through registration and prices of biological agents for cancer therapy that were authorized for sale in the last 5 years in Brazil, Colombia, and Mexico. The United States of America (USA), where most of these products are developed, and Spain, a country of the European Community with access to these supplies, will be used as bases for comparison of these Latin American countries.

\section{Materials and Methods}

The sample was limited to biological drugs used for cancer treatment registered in the last 5 years. Brazil, Colombia, and Mexico were chosen because of better access to their health agencies' databases, allowing a comparison with the USA and Spain. Availability in the market through registration was the first aspect measured related to access. A registration time of less than one year, since the first registration identified, was acceptable. As the registration systems were quite different, only the dates available for the Latin American countries and Spain were compared, with USA as a reference.

The regulatory agencies'e-websites were consulted for the survey of drugs registered between January 1, 2014, and February 20, 2019 (Agência Nacional de Vigilância Sanitária - Anvisa, from Brazil; Instituto Nacional de Vigilância de Medicamentos y Alimentos- Invima, from Colombia; and Comisión Federal para la 
Protección contra Riesgos Sanitarios - Cofepris, from Mexico) (ANVISA, 2019a; COFEPRIS, 2019a; INVIMA, 2019). All drugs with marketing authorization in that period were analyzed (BRASIL, 1976). In Spain and the USA, renewal is not required, and thus, data from the first registration were obtained. Starting dates for commercialization were obtained from the Consejo General de Colegios Oficiales de Farmacéuticos (Official Pharmacists Associations General Council) (Spain) and the Food and Drug Administration - FDA (CGCOF, 2019; FDA, 2018).

A list of products was established based on drug authorization records of each of the countries analyzed. To obtain this information for Brazil, the Anvisa website was consulted using filters for "biological drugs" and "antineoplastic drugs" (ANVISA, 2019a). For Colombia, medications with health registrations in force, by active principle, within the period (2014-2019) (INVIMA, 2019) were examined. The data for registered drugs were collected for these three countries and later compared with data from US and Spanish health agencies. Although the subject of this study was cancer, immunostimulants were not included because only a few are indicated for oncology treatment.

The second aspect related to access that was measured was price. Pharmaceutical retail prices were sought in the official databases of the studied countries. In Brazil, pharmacies, drugstores, laboratories, distributors, and importers cannot charge prices for medicines higher than allowed by the Medicine Market Regulatory Chamber (CMED - Câmara de Regulação do Mercado de Medicamentos). The list of maximum prices allowed for the sale of medicines is available for consumer consultation and is updated monthly. The retail price and the February 2019 list were used (ANVISA, 2019b).

For Colombia, the database included all drugs, which at the time, had a maximum selling price, with a reference price according to the provisions of the National Commission for Medicine and Medical Device Prices (Comisión Nacional de Precios de Medicamentos y Dispositivos Médicos).Commercial prices for market transactions, provided in an official list of regulated reference prices relative to February 2019 (MINISTERIO DE LA SALUD Y PROTECCION SOCIAL, 2019), were used. For Mexico, drug prices were determined using a government website and a site recommended by the government. Many prices, referring to June 30, 2018, were on a retail price list made available by the country's government (GOVERNO DO MÉXICO, 2019; SAN PABLO FARMACIA, 2019). 
The prices of the biological agents in these countries were compared with those found in the US market, the largest in the world, and the Spanish market, a European country with access to high-cost treatments. In the USA, market prices were obtained from Drugs.com (DRUGS.COM, 2019), and those from Spain were obtained from the Consejo General de Colegios Oficiales de Farmacéuticos (Official Pharmacists Associations General Council, Spain) (CGCOF, 2019). In the case of Spain these data correspond to drugs that already have a national positive decision on price and reimbursement and hence, they can be prescribed by clinicians (they are not only registered but authorized to be marketed). As the drug samples differed in presentation and concentration, all samples were standardized by unit of presentation. For injectable solutions, the unit analyzed was $\mathrm{ml}$ and for noninjectable, capsule. Prices were calculated based on the lowest value found in the market, whether branded or generic. All prices were converted into US dollars (US\$) using the Central Bank of Brazil converter (BRAZIL CENTRAL BANK, 2019).

Furthermore, drug prices were also compared among Latin American countries, the USA, and Spain using purchasing power/purchasing power parity (PP or PPP) or only PPP (WORLD BANK, 2019a). This tool eliminates distortions generated by the different price levels between countries (WORLD BANK, 2019a, 2019b). For example, someone receives $\mathrm{R} \$ 1,000.00$ in Brazil and wants to spend it shopping in New York (USA). Using the PPP, in New York, US\$ 455.83 would be necessary to purchase the same items that could be purchased in Brazil with $\mathrm{R} \$ 1,000.00$, which is approximately US\$262.39, based on the exchange rate of March 18, 2019, reflecting the lower Brazilian purchasing capacity due to the lower per capita income (WORLD BANK, 2019b).

According to Iyengar and his collaborators (2016), the price of a drug in different countries should, in theory, be the same when expressed in a common currency, such as the PPP exchange rates. PPP adjustment is important when comparing drug prices between countries to establish differences in the PP of goods and services (WORLD BANK, 2019a, 2019b). PPP values for 2017 were used (WORLD BANK, 2019a). This study did not require approval from the institutional review board or informed consent because it is based on public data and does not involve patient records. 


\section{Results}

Among the 25 drugs listed, none is registered and appears on all official lists from the three Latin American countries. Five biological agents are available in two countries concomitantly, and the others are officially available in one country. The USA and Spain have 25 and 22 biological agents registered, respectively.

Among the 21 biological cancer drugs authorized in Brazil between 2014 and 2019, three had outdated registrations, and one was designated a biosimilar. The sample consisted of 16 drugs. Two were approved for breast cancer treatment (12.5\%), seven for hematological tumors (43.75\%), and the remaining seven $(43.75 \%)$ for colorectal cancer, gastric cancer, urothelial cancer, melanoma, sarcoma, and renal carcinoma (ANVISA, 2019a).

In Colombia, of 16 registered drugs, three were in the process of registration renewal (aflibercept) or had exceeded the expiry date (ipilimumab and panitumumab), and four were registered before 2014 (bevacizumab, cetuximab, rituximab, and trastuzumab) (INVIMA, 2019). In the final sample of nine biological agents, five were registered for hematological malignancies, two for lung cancer, one for breast cancer, and one for melanoma.

The Federal Commission for the Protection against Sanitary Risk (Cofepris), which is responsible for drug registration in Mexico, approved 5 biological drugs from 2014 to February 2019. Drugs registered before 2014 were not included in the sample but their registrations were valid and the drugs were being marketed in the country (bevacizumab, cetuximab, ofatumumab, panitumumab, trastuzumab and trastuzumab emtansine) (INVIMA, 2019). Four biological agents were registered: one for colorectal cancer treatment, one for breast cancer, one for hematological malignancies, and one for advanced gastric cancer. The USA and Spain, after marketing approval, do not require registration revalidation. All drugs included in the Latin American samples were searched in databases from these two countries (CGCOF, 2019; FDA, 2018). Table 1 presents these agents, their main indications, and their registration dates in each of the analyzed countries. 
Table 1. Registration dates for biological drugs approved for cancer from 2014 to 2019 in Brazil, Colombia and Mexico compared with Spain and USA

\begin{tabular}{|c|c|c|c|}
\hline Active principle & Main indication (FDA) & Registration date & Country \\
\hline Aflibercept & metastatic colorectal cancer (mCRC) & $\begin{array}{l}24 / 03 / 2014 \\
18 / 11 / 2011 \\
02 / 11 / 2016\end{array}$ & $\begin{array}{l}\text { Spain } \\
\text { USA } \\
\text { Mexico }\end{array}$ \\
\hline Afatinib & $\begin{array}{l}\text { locally advanced or metastatic non-small- } \\
\text { cell lung cancer }\end{array}$ & $\begin{array}{l}13 / 06 / 2014 \\
12 / 07 / 2013 \\
01 / 01 / 2015\end{array}$ & $\begin{array}{l}\text { Spain } \\
\text { USA } \\
\text { Colombia }\end{array}$ \\
\hline Asparaginase & Acute leukemia, particularly lymphocytic & $\begin{array}{l}14 / 01 / 2019 \\
01 / 08 / 2002\end{array}$ & $\begin{array}{l}\text { Brazil } \\
\text { USA }\end{array}$ \\
\hline Atezolizumab & $\begin{array}{l}\text { urothelial carcinoma (UC), non-small cell } \\
\text { lung cancer (NSCLC) }\end{array}$ & $\begin{array}{l}09 / 10 / 2017 \\
18 / 05 / 2016 \\
21 / 03 / 2018\end{array}$ & $\begin{array}{l}\text { Brazil } \\
\text { USA } \\
\text { Spain }\end{array}$ \\
\hline Avelumab & $\begin{array}{l}\text { metastatic Merkel cell carcinoma (MCC), } \\
\text { urothelial carcinoma (UC), renal cell } \\
\text { carcinoma (RCC) }\end{array}$ & $\begin{array}{l}04 / 06 / 2018 \\
01 / 09 / 2018 \\
23 / 03 / 2017\end{array}$ & $\begin{array}{l}\text { Brazil } \\
\text { Spain } \\
\text { USA }\end{array}$ \\
\hline Bevacizumab & $\begin{array}{l}\text { metastatic colorectal cancer, non-small } \\
\text { cell lung cancer, glioblastoma, and } \\
\text { metastatic renal cell cancer }\end{array}$ & $\begin{array}{l}11 / 02 / 2019 \\
11 / 07 / 2005 \\
26 / 02 / 2004\end{array}$ & $\begin{array}{l}\text { Brazil } \\
\text { Spain } \\
\text { USA }\end{array}$ \\
\hline Blinatumomab & $\begin{array}{l}\text { acute lymphoblastic leukemia (ALL) in } \\
\text { adults and children }\end{array}$ & $\begin{array}{l}17 / 04 / 2017 \\
01 / 01 / 2018 \\
03 / 12 / 2014\end{array}$ & $\begin{array}{l}\text { Brazil } \\
\text { Colombia } \\
\text { USA }\end{array}$ \\
\hline $\begin{array}{l}\text { Brentuximab } \\
\text { vedotin }\end{array}$ & $\begin{array}{l}\text { Recurrent or refractory Hodgkin's } \\
\text { lymphoma (HL) }\end{array}$ & $\begin{array}{l}01 / 09 / 2014 \\
12 / 02 / 2014 \\
01 / 08 / 2014 \\
19 / 08 / 2011\end{array}$ & $\begin{array}{l}\text { Brazil } \\
\text { Colombia } \\
\text { Spain } \\
\text { USA }\end{array}$ \\
\hline Carfilzomib & relapsed or refractory multiple myeloma & $\begin{array}{l}01 / 01 / 2015 \\
01 / 05 / 2018 \\
20 / 07 / 2012 \\
\end{array}$ & $\begin{array}{l}\text { Colombia } \\
\text { Spain } \\
\text { USA }\end{array}$ \\
\hline Cetuximab & metastatic colorectal cancer & $\begin{array}{l}17 / 05 / 2007 \\
12 / 02 / 2004\end{array}$ & $\begin{array}{l}\text { Spain } \\
\text { USA }\end{array}$ \\
\hline Crizotinib & $\begin{array}{l}\text { positive advanced non-small cell lung } \\
\text { cancer (NSCLC) }\end{array}$ & $\begin{array}{l}24 / 07 / 2014 \\
01 / 12 / 2013 \\
26 / 08 / 2011\end{array}$ & $\begin{array}{l}\text { Colombia } \\
\text { Spain } \\
\text { USA }\end{array}$ \\
\hline Elotuzumab & multiple myeloma & $\begin{array}{l}18 / 12 / 2017 \\
01 / 01 / 2018 \\
30 / 11 / 2015\end{array}$ & $\begin{array}{l}\text { Brazil } \\
\text { Spain } \\
\text { USA }\end{array}$ \\
\hline
\end{tabular}




\begin{tabular}{|c|c|c|c|}
\hline Active principle & Main indication (FDA) & Registration date & Country \\
\hline Ibrutinib & Chronic lymphocytic limphoma & $\begin{array}{l}19 / 02 / 2015 \\
01 / 01 / 2016 \\
13 / 11 / 2013\end{array}$ & $\begin{array}{l}\text { Colombia } \\
\text { Spain } \\
\text { USA }\end{array}$ \\
\hline $\begin{array}{l}\text { Inotuzumab } \\
\text { ozogamicin }\end{array}$ & acute lymphoblastic leukemia (ALL) & $\begin{array}{l}21 / 01 / 2019 \\
17 / 08 / 2017\end{array}$ & $\begin{array}{l}\text { Brazil } \\
\text { USA }\end{array}$ \\
\hline Nivolumab & $\begin{array}{l}\text { advanced melanoma (unresectable or } \\
\text { metastatic) and locally advanced or } \\
\text { metastatic non-small-cell lung cancer } \\
\text { (NSCLC) }\end{array}$ & $\begin{array}{l}04 / 04 / 2016 \\
01 / 01 / 2016 \\
01 / 01 / 2016 \\
22 / 12 / 2014\end{array}$ & $\begin{array}{l}\text { Brazil } \\
\text { Colombia } \\
\text { Spain } \\
\text { USA }\end{array}$ \\
\hline Obinutuzumab & chronic lymphocytic leukemia (CLL) & $\begin{array}{l}23 / 03 / 2015 \\
01 / 01 / 2014 \\
19 / 10 / 2015 \\
01 / 11 / 2013\end{array}$ & $\begin{array}{l}\text { Brazil } \\
\text { Colombia } \\
\text { Spain } \\
\text { USA }\end{array}$ \\
\hline Ofatumumab & chronic lymphocytic leukemia (CLL) & $\begin{array}{l}01 / 07 / 2014 \\
26 / 10 / 2009\end{array}$ & $\begin{array}{l}\text { Spain } \\
\text { USA }\end{array}$ \\
\hline Olaratumab & soft tissue sarcoma (STS) & $\begin{array}{l}26 / 12 / 2017 \\
01 / 11 / 2017 \\
19 / 10 / 2016\end{array}$ & $\begin{array}{l}\text { Brazil } \\
\text { Spain } \\
\text { USA }\end{array}$ \\
\hline Panitumumab & carcinoma of the colon or rectum & $\begin{array}{l}20 / 04 / 2015 \\
18 / 12 / 2007 \\
27 / 09 / 2006\end{array}$ & $\begin{array}{l}\text { Brazil } \\
\text { Spain } \\
\text { USA }\end{array}$ \\
\hline Pegaspargase & acute lymphoblastic leukemia (ALL) & $\begin{array}{l}26 / 02 / 2018 \\
01 / 06 / 2017 \\
24 / 07 / 2006 \\
\end{array}$ & $\begin{array}{l}\text { Brazil } \\
\text { Spain } \\
\text { USA }\end{array}$ \\
\hline Pertuzumab & $\begin{array}{l}\text { metastatic HER2-positive breast } \\
\text { cancer, metastatic or locally recurrent, } \\
\text { nonresectable }\end{array}$ & $\begin{array}{l}15 / 06 / 2014 \\
08 / 06 / 2012 \\
16 / 05 / 2017\end{array}$ & $\begin{array}{l}\text { Spain } \\
\text { USA } \\
\text { Mexico }\end{array}$ \\
\hline Ramucirumab & $\begin{array}{l}\text { advanced gastric cancer or } \\
\text { gastroesophageal junction } \\
\text { adenocarcinoma }\end{array}$ & $\begin{array}{l}22 / 02 / 2016 \\
01 / 12 / 2015 \\
21 / 04 / 2014 \\
04 / 08 / 2015\end{array}$ & $\begin{array}{l}\text { Brazil } \\
\text { Spain } \\
\text { USA } \\
\text { Mexico }\end{array}$ \\
\hline Rituximab & $\begin{array}{l}\text { low-grade non-Hodgkin lymphoma. } \\
\text { Chronic lymphocytic leukemia }\end{array}$ & $\begin{array}{l}07 / 09 / 1998 \\
26 / 11 / 1997 \\
15 / 02 / 2017\end{array}$ & $\begin{array}{l}\text { Spain (17) } \\
\text { USA (18) } \\
\text { Mexico (15) }\end{array}$ \\
\hline Trastuzumab & $\begin{array}{l}\text { metastatic breast cancer, early HER2- } \\
\text { positive cancer, and advanced gastric } \\
\text { cancer }\end{array}$ & $\begin{array}{l}18 / 12 / 2017 \\
21 / 11 / 2000 \\
25 / 09 / 1998\end{array}$ & $\begin{array}{l}\text { Brazil } \\
\text { Spain } \\
\text { USA }\end{array}$ \\
\hline
\end{tabular}




\begin{tabular}{l|c|c|l}
\hline Active principle & Main indication (FDA) & Registration date & \multicolumn{1}{c}{ Country } \\
\hline Trastuzumab & metastatic HER2-positive breast cancer & $06 / 01 / 2014$ & Brazil \\
emtansine & & $07 / 01 / 2014$ & Colombia \\
& & $15 / 06 / 2015$ & Spain \\
& & $22 / 02 / 2013$ & USA \\
\hline
\end{tabular}

Source: Prepared by the authors with data from (ANVISA, 2019a; COFEPRIS, 2019b; INVIMA, 2019). * Cetuximab and panitumumab have an authorization date and non-commercialization in Spain (CGCOF, 2019).

Brazilian registrations were concentrated in 2017, when five drugs were approved for commercialization (29.4\%). In Colombia, the most approvals occurred in 2014, with four drugs (44.4\%) approved, and in Mexico, the most approvals occurred in 2017 (50.0\%) (ANVISA, 2019b; COFEPRIS, 2019a; INVIMA, 2019). In the USA, $70 \%$ of the registrations occurred between 1990 and 2013, while Spain, during this same period, authorized the sale of $32 \%$ of cancer agents in the sample.

USA had the highest number of first registrations (92.6\%). The mean difference between the time of release in the USA and in the other countries was analyzed as the "availability" of these supplies; Spain, Brazil, Colombia, and Mexico had the following mean values: 900, 1914, 595, and 2776 days, respectively. On average, after the launch of a biological agent in the US market, registration occurs in Spain after 990 days and in Colombia after 564 days. Table 2 shows drug prices in dollars and in PPP, per presentation unit of the pharmaceutical form in which they were found in the markets analyzed. 


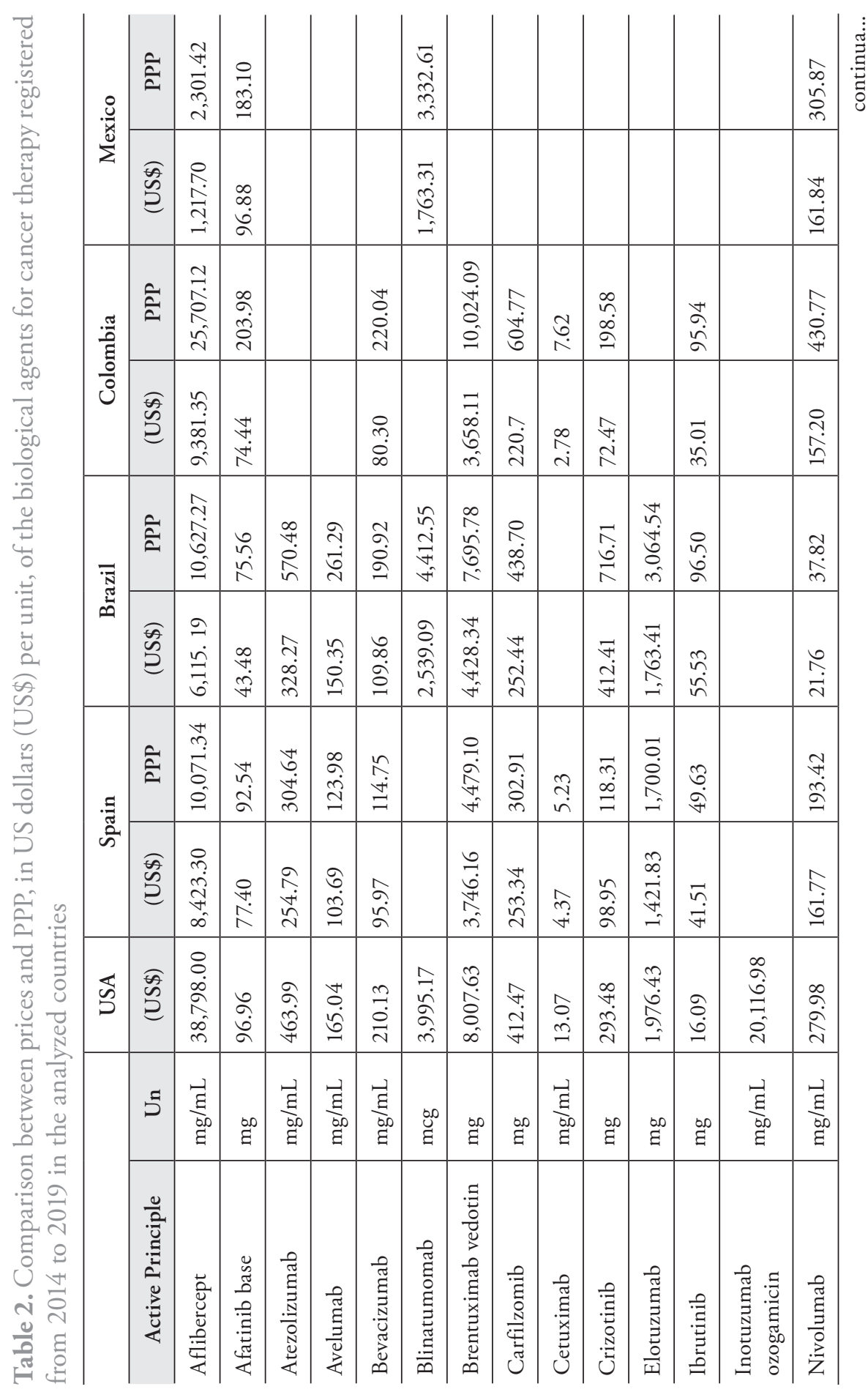




\begin{tabular}{|c|c|c|c|c|c|c|c|c|c|c|c|c|}
\hline \multirow{2}{*}{ 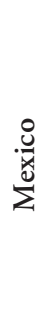 } & $\hat{\overrightarrow{\mathrm{a}}}$ & & & & & & 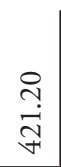 & & $\begin{array}{l}\stackrel{0}{0} \\
\text { ర్ర }\end{array}$ & & & \multirow{11}{*}{ 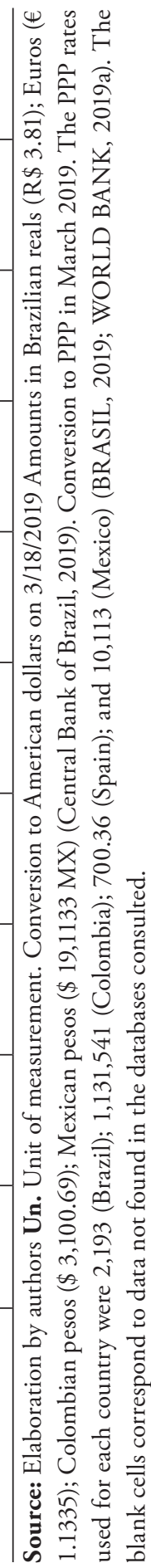 } \\
\hline & 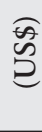 & & & & & & 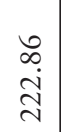 & & ஸे & & & \\
\hline \multirow{2}{*}{ 茪 } & $\hat{\overrightarrow{\mathrm{a}}}$ & $\begin{array}{l}\cong \\
\stackrel{ }{=}\end{array}$ & & & $\begin{array}{l}\stackrel{\infty}{\infty} \\
\stackrel{\vec{\sim}}{\sim}\end{array}$ & & & & 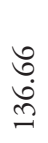 & $\begin{array}{l}0 \\
\stackrel{0}{0} \\
\dot{0} \\
\stackrel{0}{+} \\
\stackrel{+}{-1}\end{array}$ & 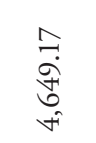 & \\
\hline & 厄્ & 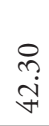 & & & $\begin{array}{l}8 \\
\stackrel{i}{+} \\
+\infty\end{array}$ & & & & $\begin{array}{l}\hat{\infty} \\
\stackrel{\sigma}{\sigma}\end{array}$ & $\stackrel{\substack{7 \\
\stackrel{+}{+}}}{\text { in }}$ & 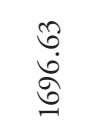 & \\
\hline \multirow{2}{*}{ 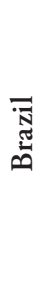 } & $\hat{\overline{\mathrm{a}}}$ & $\begin{array}{l}\tilde{n} \\
\text { तิ } \\
\text { ते }\end{array}$ & & $\stackrel{+}{\stackrel{+}{+}}$ & $\begin{array}{l}\text { If } \\
\stackrel{0}{\circ} \\
\stackrel{+}{ \pm}\end{array}$ & ?ִ & $\begin{array}{l}\curvearrowright \\
\hat{n} \\
\tilde{n}\end{array}$ & $\begin{array}{l}\vec{n} \\
\stackrel{+}{\sim}\end{array}$ & $\begin{array}{l}\text { तु } \\
\stackrel{\Xi}{\Xi}\end{array}$ & $\begin{array}{l}\infty \\
\infty \\
i \\
\infty \\
\infty \\
-1\end{array}$ & $\begin{array}{l}0 \\
i \\
0 \\
\stackrel{n}{n} \\
\dot{n}\end{array}$ & \\
\hline & & 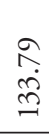 & & $\begin{array}{l}\vec{\sigma} \\
\stackrel{\leftrightarrow}{\psi}\end{array}$ & $\begin{array}{l}\text { N1 } \\
\infty \\
\infty\end{array}$ & $\begin{array}{l}\stackrel{m}{\leftrightarrow} \\
\stackrel{+}{f} \\
m\end{array}$ & 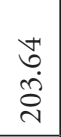 & $\begin{array}{l}\widetilde{\sigma} \\
\infty \\
\infty\end{array}$ & $\begin{array}{l}\infty \\
\infty \\
\infty \\
\infty\end{array}$ & \begin{tabular}{l}
\multirow{f}{f}{} \\
$\dot{0}$ \\
$\infty$ \\
0 \\
-
\end{tabular} & $\begin{array}{l}\stackrel{n}{\sim} \\
\stackrel{0}{\infty} \\
\infty \\
-\end{array}$ & \\
\hline \multirow{2}{*}{ ڤี } & $\hat{\overline{\mathbf{A}}}$ & $\underset{\stackrel{+}{r}}{\stackrel{\sim}{r}}$ & $\stackrel{\bar{\infty}}{\stackrel{\infty}{n}}$ & $\begin{array}{l}\widetilde{\Omega} \\
\dot{F}\end{array}$ & $\begin{array}{l}\hat{o} \\
\infty \\
\infty\end{array}$ & 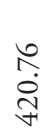 & $\begin{array}{l}\stackrel{\curvearrowleft}{\forall} \\
\stackrel{\sim}{\curvearrowright}\end{array}$ & $\underset{\infty}{\infty}$ & $\begin{array}{l}\tilde{\sigma} \\
\dot{0} \\
\tilde{n}\end{array}$ & $\begin{array}{l}\text { : } \\
\dot{0} \\
\dot{\infty}\end{array}$ & 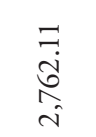 & \\
\hline & ? & $\begin{array}{l}\hat{\Xi} \\
\underset{J}{J}\end{array}$ & $\begin{array}{l}\stackrel{0}{a} \\
\stackrel{\sigma}{\sigma}\end{array}$ & $\begin{array}{l}\tilde{\delta} \\
\stackrel{+}{m}\end{array}$ & $\begin{array}{l}\text { ô } \\
\text { ते }\end{array}$ & $\begin{array}{l}\bar{\sigma} \\
\bar{n} \\
\tilde{n}\end{array}$ & $\begin{array}{l}\stackrel{\infty}{f} \\
\stackrel{\mathscr{f}}{\sim}\end{array}$ & $\underset{\hat{\sigma}}{\stackrel{\hat{\sigma}}{*}}$ & $\begin{array}{l}\stackrel{0}{\circ} \\
\stackrel{\infty}{\infty} \\
\stackrel{N}{N}\end{array}$ & $\underset{\hat{\sigma}}{\stackrel{ }{*}}$ & $\begin{array}{l}n \\
\overrightarrow{0} \\
\vec{n} \\
\tilde{n}\end{array}$ & \\
\hline 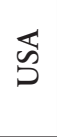 & ? & $\begin{array}{l}\hat{\sigma} \\
\infty \\
\infty \\
0\end{array}$ & $\begin{array}{l}\infty \\
\stackrel{\infty}{J} \\
\underset{J}{*}\end{array}$ & $\stackrel{n}{\stackrel{n}{n}}$ & $\begin{array}{l}\hat{\sigma} \\
\stackrel{\hat{\imath}}{\sim}\end{array}$ & 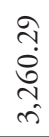 & $\begin{array}{l}\stackrel{ }{+} \\
\stackrel{+}{+} \\
\hat{n}\end{array}$ & 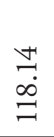 & 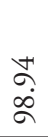 & $\begin{array}{l}\stackrel{+}{+} \\
\hat{\tilde{\sigma}} \\
\stackrel{-}{-1}\end{array}$ & $\begin{array}{l}\infty \\
\infty \\
\stackrel{\infty}{\sim} \\
\stackrel{n}{n}\end{array}$ & \\
\hline & sี & $\frac{\vec{\Xi}}{\underline{0.0}}$ & $\frac{\vec{g}}{00}$ & $\frac{\vec{\xi}}{\stackrel{0}{00}}$ & $\frac{\vec{\xi}}{\stackrel{0}{00}}$ & $\frac{\vec{g}}{\supset}$ & $\begin{array}{l}\overrightarrow{\underline{g}} \\
\overrightarrow{00} \\
\underline{q}\end{array}$ & $\begin{array}{l}\vec{\xi} \\
\stackrel{\vec{g}}{00} \\
\Xi\end{array}$ & $\frac{\vec{\Xi}}{\underline{0}}$ & $\stackrel{0}{a}$ & $\stackrel{\infty}{\Xi}$ & \\
\hline & 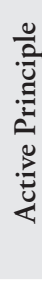 & 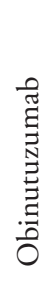 & 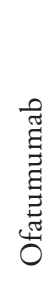 & 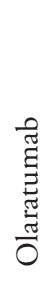 & 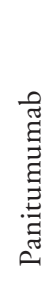 & 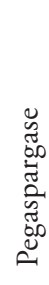 & 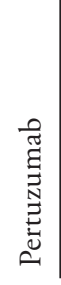 & 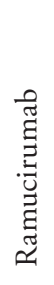 & 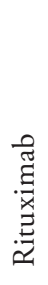 & 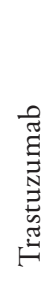 & 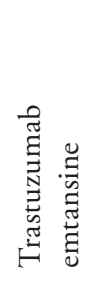 & \\
\hline
\end{tabular}


Regarding the prices of biological agents for cancer therapy, the USA is the most expensive country. When compared to the three studied Latin American countries, Spain behaves in an intermediate manner, that is, its prices are neither too high nor too low. Mexico has the highest prices for biological cancer drugs in Latin America, followed by Brazil. When US prices are compared to Spanish prices, Spain has more inexpensive drugs, except for ibrutinib and rituximab.

Prices were also compared among countries using PPP. Purchasing capacity changes in relation to the prices indicated above. In the previous analysis, the USA had higher prices for biological agents for cancer therapy, but not when considering PPP. US prices are lower than the PPP values for Latin American countries, except for aflibercept, ofatumumab and pegaspargase. The PPP for Spain is lower than that for Latin America and the US for almost all drugs (only rituximab has a higher PPP value). When Brazil is compared vis a vis the other countries, its PPP values are higher than those of US prices and the PPP for Spain. Brazil and Colombia have similar PPP values, considering the relative number of drugs in each of these countries. If the absolute number is analyzed, Brazil, with a greater number of drugs approved in the period, has a higher PPP than that of Colombia but a PPP lower than that of Mexico. Colombia, Brazil, and Mexico have PPP values higher than those of the US and Spain. Mexico has the highest PPP, considering the number of drugs analyzed.

To avoid distortions, the drugs marketed in each of the countries and their North American counterparts were analyzed. In addition, Aflibertcept is excluded from the evaluation, whose value in the US reaches 15 times that practiced in Mexico. For Spain, the 20 drugs negotiated are on average $40 \%$ cheaper than in the US, while in Mexico, the 5 existing products are 10\% cheaper. In Brazil, the price of 20 medicines is almost the same, being only $3 \%$, on average, cheaper. In Colombia, this relationship is reversed, where 13 drugs sold are $26 \%$ more expensive.

\section{Discussion}

The main objective of this study was to compare access among Latin American markets to biological agents for cancer therapy that have become available in the last 5 years. From this perspective, searches were conducted in the USA, where most of the first registrations occur, and Spain, an intermediate country regarding authorization time (PUIG-JUNOY, 2013; VEGA, 2002). 
In Brazil, the date of first registration on the Anvisa website is not always reliable (ANVISA, 2019a). In Mexico, there have been approvals previous to those by the FDA, such as the case of trastuzumab and bevacizumab (COFEPRIS, 2019a). It is possible that these drugs have been approved for other uses in the country, and their registration has been maintained. Medications with 2 years of validity (rituximab) was found. In 2007, drug registration renewal was reviewed in the country. Authorization to market organic products whose active ingredient is not registered in the country, but with authorization to sell in the European Union, Switzerland, the USA, Canada, and Australia, can be obtained (COFEPRIS, 2019b). These differences in legislation may perhaps explain the different valid registrations among Latin American countries.

There are still some drugs that do not appear in the sample, because they are under registration renewal, such as Aflibercept in Colombia and Rituximab in Brazil; mistakenly suggesting that they are not present in these markets. Regarding their availability, when considering only the 18 drugs approved after 2014 and after their launch in the US market, the countries analyzed take, on average, 1.6 to 7.6 years to register the products in their markets. None of the countries analyzed, including Spain, provides oncological drugs within a period of less than one year. A more detailed analysis would identify biases, such as the absolute number of drugs surveyed in each market, which should be considered because it distorts the results, in addition to errors related to drug registration.

In 2016, new drug registration, generic drug registration, and biosimilar drug registration by Anvisa took 632 days, 1062 days, and 1225 days, respectively. Streamlining release processes through shorter deadlines for drug registration was a charge of the pharmaceutical industry and a challenge for the agency. In 2018, the average total time to grant drug registration was 188 days for generic and biosimilar drugs, 276 days for new drugs and 356 days for innovators, considering the petitions filed since April 2017 (ANVISA, 2018; BRASIL, 2016). In 2015, new drug approvals led to an average of 478 days of use by the European Medicines Agency, compared to 304 days by the US FDA (KUBLER, 2018).The agility in the approval process of these agents is linked to their clinical attributes and invades issues related to public health, such as economic, political and social ones (INTERFARMA., 2013; KIM; PRASAD, 2015). 
The blank cells in Table 2 may be related to recent approvals in the market (e.g., inotuzumab ozogamicin, registered in 2019 in Brazil), the non-commercialization of the product on the market or the absence of distributors. Given the availability of information on drug registration in these countries, it is not possible to address the availability of theseagents in Latin American markets. These trends and characteristics of marketing approval would provide valuable information for the pharmaceutical industry as well as for prescribers and patients (MARKETRESEARCH.COM., 2019; MARTELL; SERMER; GETZ, 2013).

Regarding biological agent prices, the blank cells in table 2 also indicate, in general, what occurs in the market of each analyzed country. Whether it is a recent approval, absence of a distributor, lack of product or nonmarketing option by the pharmaceutical industry, the price or the drug was not found.

The US market has high drug prices (MARKETRESEARCH.COM., 2019; MOREL; MCGUIRE; MOSSIALOS, 2011). Brazil and Colombia may have had lower prices because the values suggested to the distributor were used instead of the final price the consumer paid. In both markets, there are regulatory mechanisms that are based on international comparisons to establish limits on sale prices (ANVISA, 2019b). In a simple comparison between countries, there is great heterogeneity, which may also be associated with distinct units of measurement and packaging. According to Álvarez and González (2018), who analyzed the prices of various classes of drugs sold in Latin America, the observed differences can be attributed to supplies costs, tariff barriers, taxes, industry regulations, market power, reimbursement systems or consumer preference. Variation between LA countries and U.S. has been attributed to differences in pricing and reimbursement regulations, type of insurance coverage, prescribing policies, and laws, among other factors (ÁLVAREZ; GONZÁLEZ, 2018). A substantial variation between cancer drug prices in Latin American countries was observed by Ruiz et al. (2017), where the annual value for the use of trastuzumab as an adjuvant for breast cancer ranged from US\$25,636 in Uruguay to US\$61,302 in Brazil in 2012.

Determining prices is another problem that these markets have. An unwritten rule provides similar prices for new products from Europe and the USA, although the lowest reference is usually taken, corresponding to nearby countries or countries with lower prices. We want to avoid a parallel market, a bitter topic for the 
pharmaceutical industry, which could lead to economic losses from selling the same drug at lower prices (VEGA, 2002).

Prices were also compared among countries using PPP. As previously mentioned, this is an important tool when relating drug prices among countries to establish differences in PP levels (BANK, 2019; “Salary Convert.", 2019). Our study failed to establish a clear standard, but the affordability of these agents by citizens of Latin American countries is similar to that of those in the US and much higher than that of Spaniards. The observed affordability trend in Mexico is higher than that in the other countries analyzed, despite the small sample. The drugs, which at first seem more inexpensive show their real purchase value when standardized by PPP.

Morel et al. (2020) found no relationship between drug prices in low- and middle-income countries and their gross domestic product. They compared the prices of high-cost drugs, including eight cancer therapies. Throughout the study period, between 2003 and 2007, 13 of the 14 middle-income countries analyzed had lower prices than did the USA, except for Mexico, where prices were similar to those practiced in the USA. The United Kingdom paid less for all drugs analyzed than did Argentina, Brazil, Paraguay and Uruguay. Iyengar et al. (2016) analyzed the price and accessibility of hepatitis $\mathrm{C}$ therapies in 30 countries, comparing them with national economic performance, estimated market size, cost in relation to total annual pharmaceutical expenditure of countries, and time one individual would need to work to pay for treatment. They converted drug prices into US\$ and adjusted them by PPP. The average price of a 12-week treatment with sofosbuvir in 26 OECD countries ranged from US\$37,729 in Japan to US\$ 64,680 in USA. The authors concluded that the current prices of these drugs are globally variable and inaccessible, adjusted by PPP.

This scenario changes when we consider the purchasing power of each country. Using GDP per capita measured in PPP (WORLD BANK, 2019a) as a proxy of this measure, for Spain, the average value is almost equal to the North American, going from $40 \%$ cheaper to $6 \%$. Brazil, Colombia and Mexico have values of $371 \%, 520 \%$ and $148 \%$, respectively. As price is a barrier to access to medicines, it can be inferred that these three countries have difficulties in ensuring that biological products reach patients who need them.

Given the high degree of innovation in the sector, new technologies are often incorporated in an accelerated manner, even before enough evidence proves their 
safety, efficacy, and effectiveness. This is associated with higher costs in relation to preexisting technologies. Knowing that resources are always finite in the face of society's demand, there is constant conflict between the use of resources and the need to choose between alternative allocations. The opportunity cost, i.e., the application of resources in certain technologies that imply the non-provision of others, should not be forgotten, especially in countries where there is still a high prevalence of morbidity and mortality from diseases of poverty (PALMER; RAFTERY, 1999).

The findings in this study should be interpreted carefully. Biological agents are just one drug class among the many existing in the countries analyzed. An attempt was made to compare similar drug prices; although some drug samples coincide with each other, they may differ in presentation, concentration, formulation, manufacturer, and time in which they are available on the market. An ideal price analysis would control all these characteristics.

The authors used "marketing authorization" as a proxy for availability of these medications, as a partial photo. A drug can have a marketing authorization, but it still has not been brought on the market. It would be interesting to have sales, commercialization data for these drugs. Another limitation is the use of an administrative database, considering its structural aspects - such as information gaps and difficulties in the codification of procedures, which restrict the possibility of developing assessments based on this information. The downscaling of the data and, in the US case, the inexistence of an official price base should also be scored. In Spain, for hospital medicines, only the ex-factory prices exist. Whether the drugs were marketed or designated as orphan drugs or for compassionate use was not analyzed. The comparison between the PP of the three Latin American countries is hampered by the heterogeneity of the markets and by the great uncertainty in the data presented. PPP is a mechanism for accounting for different costs related to goods, when carrying out comparative analysis of expenses, in different countries, but it has some limitations. The theory shows a direct link between the purchasing power and exchange rate and ignores many other factors of exports and imports involved behind the operation. PPP's theory overlooks the influence of demand and supply factors in foreign exchange and holds good in the long run. The theory involves a practical difficulty of measuring the true purchasing power of a currency. But the fact that perfect measurement is unattainable, does not mean that nothing can be done (MOON et al., 2010). 
The scarcity of studies of this nature, the difficulty in analyzing information from such different databases with so many peculiarities, and the great potential of available data for health services are noteworthy.

\section{Conclusion}

This study compared drug prices between Brazil, Colombia, Mexico, the USA, and Spain. The registration time differs in the countries analyzed, pointing to a problem in access or lack of equity, in comparative terms. It is believed that a reduction in waiting time for finalizing the analysis of drug registration requests would result in attaining new treatments faster and new oncology therapeutic protocols and guidelines. In turn, the economic effort these countries make is much higher than that of the US and Spanish efforts.

The PPP strategy was needed to ensure a better understanding of access to pharmaceuticals. Countries with higher PP have higher prices, and those with lower PP have lower prices. It was noticed that there is a differentiated pricing policy applied by the pharmaceutical industry, where they maximize their profits (Ramsey's price). The industry defines its prices together with governments and sells its products at a higher price in relation to a perfectly competitive market structure. Considering these results, if the prices of these biological agents for cancer therapy could be lower, access would be increased in Latin American countries, as would the monopolist income in general. The differences noted are greater when converted into PP units.

Studies such as these are useful for understanding the price profile of innovative pharmacological agents for different markets and health systems, helping to establish research priorities, registration, and evaluation. Diseases that have high average individual costs or system costs, for which there are different therapeutic strategies, can be priority targets for evaluation. The results presented herein may serve as a basis for the composition of more robust studies on the subject. Access to missing data, both in terms of registration and prices, may change the direction of the results. ${ }^{1}$

\section{References}

AGÊNCIA NACIONAL DE VIGILÂNCIA SANITÁRIA. ANVISA. Detailed information on reducing time to finalize review of drug registration applications in Brazil. Disponível em: <http://portal.anvisa.gov.br/rss//lasset_publisher/Zk4q6UQCj9Pn/content/id/4801017>. Acesso em: 18 mar. 2019. 2018 
AGÊNCIA NACIONAL DE VIGILÂNCIA SANITÁRIA. ANVISA. Consultation of drug registration. Disponível em: <https://consultas.anvisa.gov.br/\#/medicamentos/>. Acesso em: 20 fev. 2019a.

AGÊNCIA NACIONAL DE VIGILÂNCIA SANITÁRIA. ANVISA. Drug price consultation. Disponível em: <http://portal.anvisa.gov.br/listas-de-precos>. Acesso em: 21 mar. 2019b.

ÁLVARES, J. et al. Access to medicines by patients of the primary health care in the Brazilian Unified Health System. Revista de Saúde Pública, v. 51, n. supl.2, p. 20s-20s, set. 2017.

ÁlVArEZ, R.; GONZÁlEZ, A. Comparative Analysis of Drug Prices in Latin America. Working Papers Series (SDT 462). [s.l: s.n.]. Disponível em: <http://www.econ.uchile.cl/ uploads/publicacion/5f3c7f0dcd767d1d119f9df8ef1e7424bdbbcba5.pdf>. 2018

ASSOCIAÇÃO DA INDÚSTRIA FARMACÊUTICA DE PESQUISA. Biological and biosimilar medicines and their regulatory norms. Patient Primer. São Paulo: Interfarma. Associação da Indústria Farmacêutica de Pesquisa, 2013.

BRASIL. PRESIDÊNCIA DA REPÚBLICA. Lei nº 6360 de 23 de setembro de 1976. Dispóe sobre a Vigilância Sanitária a que ficam sujeitos os Medicamentos, as Drogas, os Insumos Farmacêuticos e Correlatos, Cosméticos, Saneantes e Outros Produtos, e dá outras Providências. [s.l: s.n.]. Disponível em: <http://www.planalto.gov.br/ccivil_03/leis/L6360. htm>. Acesso em: 20 mar 2020.

BRASIL. SENADO FEDERAL. Federal Senate. Disponível em: <https://www12.senado. leg.br/noticias/materias/2016/03/09/necessidade-de-agilizar-registro-de-medicamentos-econsenso-em-debate-na-cas>. Acesso em: 18 mar. 2019.

BRAZIL CENTRAL BANK. Currency converter. Disponível em: <https://www.bcb.gov.br/ conversao >. Acesso em: 20 mar. 2019.

CHARY, K. V.; PANDIAN, K. Accelerated approval of drug: ethics versus efficacy. Indian Journal of Medical Ethics, v. 2, n. 4, p. 244-247, 2017.

COMISIÓN FEDERAL PARA LA PROTECCIÓN CONTRA RIESGOS SANITARIOS. COFEPRIS. Consultation of drug registration. Disponível em: <http://tramiteselectronicos02. cofepris.gob.mx/BuscadorPublicoRegistrosSanitarios/BusquedaRegistroSanitario.aspx >. Acesso em: 21 fev. 2019a.

COMISIÓN FEDERAL PARA LA PROTECCIÓN CONTRA RIESGOS SANITARIOS. COFEPRIS. Sanitary registration of biological product whose active ingredient is not registered in Mexico. Disponível em: <https://www.gob.mx/tramites/ficha/registro-sanitario-de-productobiologico-cuyo-ingrediente-activo-no-esta-registrado-en-mexico/COFEPRIS4000>. Acesso em: 21 fev. $2019 b$ 
CONSEJO GENERAL DE COLEGIOS OFICIALES DE FARMACÉUTICOS. CGCOF. Drug price finder. Disponível em: <https://botplusweb.portalfarma.com/botplus.aspx>. Acesso em: 20 mar. 2019.

CONSEJO GENERAL DE COLEGIOS OFICIALES DE FARMACÉUTICOS (ESPAÑA). CGCOF. Buscador de preços de medicamentos. Disponível em: <https://botplusweb. portalfarma.com/botplus.aspx.>. Acesso em: 20 mar. 2019.

DAVIS, C. et al. Availability of evidence of benefits on overall survival and quality of life of cancer drugs approved by European Medicines Agency: retrospective cohort study of drug approvals 2009-13. BMJ, p. j4530, out. 2017.

DRUGS.COM. Buscador de preços de medicamentos. Disponível em: <https://www.drugs. com/search.php?searchterm=Lartruvo\&a=1>. Acesso em: 20 mar. 2019.

FERRARIO, A. Determinants of utilisation differences for cancer medicines in Belgium, Scotland and Sweden. The European Journal of Health Economics, v. 18, n. 9, p. 1095 $1105,2017$.

GOVERNO DO MÉXICO. List of maximum selling prices of medicines. Disponível em: <https:// www.economia.gob.mx/files/transparencia/focalizada/Lista_de_Precios_Máximos_de_Venta_ al_Público_de_medicamentos_de_patente_vigente_05072018.pdf>. Acesso em: 12 mar. 2019.

GRAMPP, G.; RAMANAN, S. Managing Unexpected Events in the Manufacturing of Biologic Medicines. BioDrugs, v. 27, p. 305-316, 2013.

INSTITUTO NACIONAL DE VIGILÂNCIA DE MEDICAMENTOS Y ALIMENTOS. INVIMA. Consultation of drug registration. Disponível em: <https://www.invima.gov.co/ consultas-registros-sanitarios>. Acesso em: 13 mar. 2019.

INTERFARMA. ASSOCIAÇÃO DA INDÚSTRIA FARMACÊUTICA DE PESQUISA. Medicamentos biológicos e biossimilares e suas normas regulatórias. Cartilha para pacientes. $1^{\mathrm{a}}$ ed. São Paulo: Interfarma. Associação da Indústria Farmacêutica de Pesquisa, 2013.

IYENGAR, S. et al. Prices, costs, and affordability of new medicines for hepatitis $\mathrm{C}$ in 30 Countries: An Economic Analysis. PLOS Medicine, v. 13, n. 5, p. e1002032,

KIM, C.; PRASAD, V. Cancer Drugs Approved on the Basis of a Surrogate End Point and Subsequent Overall Survival. JAMA Internal Medicine, v. 175, n. 12, p. 1992, dez. 2015.

KUBLER, P. Fast-tracking of new drugs : getting the balance right. Australian Prescriber, v. 41, n. 4, p. 98-99, 2018.

MARKETRESEARCH.COM. The Growing Pharmaceuticals Market: Expert Forecasts and Analysis. Disponível em: <https://blog.marketresearch.com/the-growing-pharmaceuticalsmarket-expert-forecasts-and-analysis>. Acesso em: 18 mar. 2019. 
MARTELL, R. E.; SERMER, D.; GETZ, K. Oncology Drug Development and Approval of Systemic Anticancer Therapy by the U.S. Food and Drug Administration. The Oncologist, v. 18, n. 1. p. 104-111, 2013.

MINISTERIO DE LA SALUD Y PROTECCION SOCIAL. Consulta ao preço de medicamentos. Disponível em: <https:/www.minsalud.gov.co/salud/MT/Paginas/listado-demedicamentos-con-precio-controlado.aspx.>. Acesso em: 12 mar. 2019.

MOON, S. et al. Purchasing Power Parity Measures: Advantages and limitations. Overseas Development Istitute, v. 7, n. I, p. 1-8, 2010.

MOREL, B. C. M.; MCGUIRE, A.; MOSSIALOS, E. The Level Of Income Appears To Have No Consistent Bearing On Pharmaceutical Prices Across Countries. Health Affairs, v. 30, n. 8, p. 1545-1552, 2011.

PALMER, S.; RAFTERY, J. Opportunity cost. BMJ, v. 318, p. 1551-1552, 1999.

PUIG-JUNOY, J. Regulamento de Preços dos medicamentos. Disponível em: <http:// pilleconomics.blogspot.com/2013/03/regulacion-de-precios-de-los.html>. Acesso em: 8 mar. 2019.

PUTHUMANA, J. et al. Availability of Investigational Medicines Through the US Food and Drug Administration's Expanded Access and Compassionate Use Programs. JAMA Network Open, v. 1, n. 2, p. e180283, 2018.

REGULATORY AFFAIRS PROFESSIONALS SOCIETY. Regulatoty focus: Accelerated Approval in Oncology: FDA Touts Successes, Responds to Criticism. Disponível em: <https:// www.raps.org/news-and-articles/news-articles/2018/3/accelerated-approval-in-oncology-fdatouts-succes>. Acesso em: 8 mar. 2019.

RUIZ, R. et al. Improving Access to High-Cost Cancer Drugs in Latin America: Mmuch to be done. Cancer, v. 123, n. 8, p. 1313-1323, 2017.

SAN PABLO FARMACIA. Drug price finder. Disponível em: <https://www.farmaciasanpablo. com.mx>. Acesso em: 20 mar. 2019.

SANTANA, R.S.; LEITE, S. N. . Priorities of clinical drug research in Brazil and the diseases of poverty. Rev Panam Salud Publica, v. 40, n. 5, p. 356-62, 2016.

SHEPHERD, W. G. Ramsey pricing Its uses and limits. Utilities Policy, v. 2, n. 4. p. $296-$ 298, 1992.

US FOOD AND DRUG ADMINISTRATION. FDA. Drugs@FDA: FDA-Approved Drugs. Disponível em: <https://www.accessdata.fda.gov/scripts/cder/daf/index.cfm>. Acesso em: 4 mar. 2019

VEGA, E. G. Mercado farmacéutico. Nuevo siglo. Economia y Salud, v. 16, n. 2, p. 9-12, 2002. 
WORLD BANK; PPP conversion factor, GDP. Disponível em: <https://data.worldbank.org/ indicator/PA.NUS.PPP?end=2018\&start=2018\&view=map $>$. Acesso em: 17 mar. 2019a.

WORLD BANK; Salary Converter. Disponível em: <http://salaryconverter.nigelb.me>. Acesso em: 21 mar. 2019b.

WORLD HEALTH ORGANIZATION, WHO; HEALTH ACTION INTERNATIONAL, H. Measuring medicine prices, availability, affordability and price components, 2nd edition. [s.l: s.n.]. Disponível em: <https://www.who.int/medicines/areas/access/OMS_Medicine_ prices.pdf>. Acesso em: 9 jan. 2020.2008

\section{Acknowledgments}

The authors declare no conflict of interest for this article. We thank the professor Cid M. M. Vianna, from Rio de Janeiro State University, for the significant suggestions and revising critically for intellectual content this text.

\section{Note}

${ }^{1}$ Gabriela B. G. Mosegui and Fernando Antońanzas Villar were involved in the concept and design, analysis, drafting and final approval of this paper. All authors agree with to be accountable for all aspects of the work. 


\section{Resumo}

\section{Disponibilidade de medicamentos biológicos contra o câncer: registro e preço no Brasil, Colômbia e México}

Este estudo busca entender a disponibilidade de medicamentos contra o câncer biológico por meio do registro e preços dos agentes biológicos utilizados na terapia do câncer e autorizados para venda nos últimos cinco anos no Brasil, Colômbia e México, comparando os dados com os dos Estados Unidos da América (EUA) e Espanha. Os sites das agências reguladoras foram consultados para medicamentos registrados entre $1^{\circ} \mathrm{de}$ janeiro de 2014 e 20 de fevereiro de 2019. Os preços dos medicamentos foram procurados nas bases de dados administrativas. Os preços também foram comparados usando a paridade do poder de compra (PPP). A comparação entre o poder de compra (PP) dos três países da América Latina é dificultada pela heterogeneidade de mercado e incerteza nos dados. Não há sincronização de registro. A diferença média entre o tempo de lançamento nos EUA e nos outros países é de 1,6 a 2,6 anos. Os EUA têm os menores valores de PPP, em comparação com os países latino-americanos estudados, mas preços mais altos. As diferenças no tempo de registro revelam problemas no acesso a medicamentos nos países latinoamericanos estudados ou falta de equidade entre os países. O esforço econômico que esses países fazem para ter acesso a esses suprimentos é muito maior do que o dos EUA e da Espanha.

> Palavras-chave: produtos biológicos; neoplasia; acesso a medicamentos essenciais e tecnologias de saúde; registro de produtos; preço do medicamento. 